\title{
Canonic Signed Digit Based Design of MULTIPLIER-LESS FIR FILTER USING SELF- ORGANIZING RANDOM IMMIGRANTS GENETIC ALGORITHM
}

\author{
Abhijit Chandra ${ }^{1}$ and Sudipta Chattopadhyay ${ }^{2}$ \\ ${ }^{1}$ Department of Electronics \& Telecommunication Engineering, Bengal Engineering and \\ Science University, Shibpur, Howrah, India \\ abhijit922@yahoo.co.in \\ ${ }^{2}$ Department of Electronics \& Telecommunication Engineering, Jadavpur University, \\ Kolkata, India \\ sudiptachat@yahoo.com
}

\begin{abstract}
Signal processing of present era is becoming more and more complex day by day. To meet the demand of modern signal processing, emphasis has been given to develop systems with minimum hardware. As a matter of fact, reduction in hardware complexity of digital filter has emerged as one of the upcoming research areas in present time. This paper highlights the design of multiplier-less finite impulse response (FIR) filter with the aid of an evolutionary optimization technique, namely Self-organizing Random Immigrants Genetic Algorithm (SORIGA). For this purpose, the coefficients of the filter have been encoded by binary and Canonic Signed Digit (CSD) number systems and subsequently optimized by means of SORIGA. Performance of the proposed filter has been analyzed in terms of its frequency and impulse response. Subsequently, hardware cost of the designed filter has been measured by means of a number of performance parameters and compared with few such existing filters from the literature in order to substantiate the efficiency of the proposed design.
\end{abstract}

\section{KEYWORDS}

Differential Evolution (DE), Finite impulse response (FIR) filter, hardware cost, multiplier-less filter, Selforganizing Random Immigrants Genetic Algorithm (SORIGA).

\section{INTRODUCTION}

In digital signal processing, use of finite impulse response (FIR) filter is more commonly favored over infinite impulse response (IIR) filter because of its number of valuable features like phase linearity, guaranteed stability and so on [1-2]. However, in a number of places where constraint on hardware is present, applicability of FIR filter is strongly challenged. This has motivated the researchers to think about FIR architecture that emphasizes on the reduction of hardware circuitry. In connection to this, signed powers of two (SPT) representation of filter coefficients is very much popular where the effect of multiplier is substituted by means of adders and delay elements only [3-7].

Design of hardware efficient digital filter has been carried out by several researchers over the last few decades. Amongst them, Lim and Parker are considered to be the pioneer in this field who had utilized the methods of integer programming for realizing discrete coefficient FIR filters [8]. Both the weighted minimax and weighted least square error criteria have been considered for the

DOI : 10.5121/ijaia.2013.4403 
design purpose. It has been shown that the achievable frequency response is superior to those obtained by simply rounding the coefficients.

A polynomial-time algorithm for designing digital filters with SPT coefficients, subject to a prescribed total number of SPT terms, has been presented in [9]. It has also been shown that the designed filter is even better than those obtained by direct quantization of the optimal filters of infinite word-length. Moreover, the polynomial-time complexity of the algorithm results in much shorter computation time for the filter design since it increases linearly with word-length unlike the other methods proposed in the literature.

The domain of circuit and system design is becoming noticeably influenced by means of a variety of intelligent optimization techniques of current interest and the design of multiplier-less digital filter is of no exception. In connection to this, an efficient genetic approach to the design of digital FIR filters with SPT coefficients has been presented in [10]. It has also been reported that the proposed genetic technique is able to attain better or comparable results to other methods. Moreover, an orthogonal genetic algorithm (OGA) approach for the design of cascade-form multiplier-less FIR filter has also been studied in the literature [11]. Simulation results, as described in the paper, demonstrate that the OGA-based method led to improvements in the stopband attenuation in both the examples considered.

More recently, design of discrete coefficient FIR filter has been made possible with the aid of a robust evolutionary optimization technique called Differential Evolution (DE) which has outperformed all the variants of GA in many applications of engineering and technology. One novel approach for quantizing the coefficients of low-pass FIR filter using Differential Evolution algorithm has been proposed in [12]. Authors have compared their outcome with a number of state-of-the-art powers-of-two filter design strategies and established the supremacy of their approach. The same problem has been addressed with the help of DE in [13] where the control parameters of DE have been judiciously adjusted with the help of a self-adaptive algorithm. Role of mutation strategies of DE for computationally efficient design of multiplier-less FIR filter has been investigated in [14] and finally the name of the best alternative has been suggested for the design process under consideration.

In this communication, we have employed Self-organizing Random Immigrants Genetic Algorithm (SORIGA) in order to realize multiplier-less FIR filter whose individual coefficients have been formulated as sums and/or differences of powers-of-two. Hardware cost of the resulting structure has been calculated and compared with some of the existing filters by means of a number of performance parameters introduced. The entire paper has been organized as follows: section 2 briefly describes the theoretical background of multiplier-less FIR filter with two possible representation of filter coefficients, namely binary and CSD. Proposed design of powersof-two filter has been formulated in section 3, followed by simulation results and analysis in section 4 . The paper ends with conclusive remarks in section 5 .

\section{Theoretical Background OF Multiplier-Less FIR Filter: BINARY \& CSD REPRESENTATION}

Finite duration impulse response (FIR) filter is being popularly used in the field of wired and wireless communication systems since long. Tap coefficients of an L-length FIR filter, which are nothing but the multiplication constants, may be represented by means of a row-vector as:

$$
h=\left[\begin{array}{llllllllll}
h_{0} & h_{1} & h_{2} & h_{3} & h_{4} & h_{5} & \ldots . . & h_{L-1}
\end{array}\right]
$$


Resulting output sequence $y(n)$ from any FIR filter can essentially be obtained by performing the convolution operation between the input sequence $x(n)$ and the impulse response coefficients $h$ of the FIR filter, as illustrated below [1-2]:

$$
y(n)=\sum_{k=0}^{L-1} h_{k} \cdot x(n-k)
$$

Computation of a single element of the output sequence $y(i), i \in Z^{+}$, thus necessitates the use for at most ' $\mathrm{L}$ ' multiplications along with ' $\mathrm{L}-1$ ' additions as observed from (2). In order to make the FIR filter more power efficient and hardware friendly, substitutions of direct multipliers by some simplified equivalent blocks has become a common practice among the practitioners.

Being motivated by the need of designing hardware efficient FIR filter, system designers have adopted several approaches to get rid of multipliers in the filter model. One such well recognized approach has been the replacement of the multipliers i.e. the filter coefficients by means of simple delay elements and adders which will not affect the filter performance significantly. The resulting impulse response coefficient of the designed filter thus may have the form:

$$
h_{i}=\sum_{j=0}^{\lambda-1} b_{i j} \cdot 2^{-j}, \quad i=0,1,2, \ldots ., L-1
$$

It can be interpreted from (3) that the individual coefficient can be treated as sum of power of two where the parameter ' $\lambda$ ' signifies how precisely these coefficients can be encoded and often known as word length (WL) of filter coefficients. The variable $b_{i j}$ in the above equation holds the key in formulating the coefficients in the sense that they either allow or reject any power of two (PT) terms $2^{-j}$ to be included into $h_{i}$. As the term $b_{i j}$ takes the binary decision of either inclusion or exclusion, it can assume values only from the binary set $\mathbb{B}=\{0,1\}$ or $\{0,-1\}$. As a matter of fact; the proper assignment of these coefficients for different values of the subscripts is a problem of optimization and may be dealt with some powerful evolutionary optimization techniques of current interest.

Canonic Signed Digit (CSD) is a unique signed digit number system which is most popularly used over binary arithmetic because of its capability in minimizing the number of non-zero binary digits and hence it can reduce the number of partial product additions in a hardware multiplier. It utilizes coefficients from a ternary set $\mathbb{T}=\{0,1,-1\}$ and thus leads to possible cancellation between powers-of-two terms [15]. For any arbitrary CSD number, adjacent CSD digits $c_{i}$ and $c_{i+1}$ can never be both non-zero i.e. $c_{i} \cdot c_{i+1}=0$; suggesting that for an $n$ bit number there can be at most $\left\lfloor\frac{n}{2}\right\rfloor$ non-zero digits [15-16]. For one $\Delta$ bit CSD number in the range $[-1,1)$; average number of non-zero bit is given by $\frac{\Delta}{3}+\frac{1}{9}+\mathbb{O}\left(2^{-\Delta}\right)[16]$. Therefore, on an average CSD numbers comprise of $33 \%$ fewer non-zero bits than two's complement numbers.

\section{DESIGN FORMULATION}

Design of multiplier-less FIR filter whose tap coefficients are encoded as sums or differences of powers of two has been felicitated in this article by means of a powerful self organizing optimization technique known as Self-organizing Random Immigrants Genetic Algorithm (SORIGA). This section briefly outlines the design formulation of FIR filter preceded by a concise discussion on SORIGA. 
International Journal of Artificial Intelligence \& Applications (IJAIA), Vol. 4, No. 4, July 2013

\subsection{Self-organizing Random Immigrants Genetic Algorithm}

Evolutionary optimization techniques had been playing major role in optimizing functions with non-linear and non-differentiable surface plane since a long time. Genetic Algorithm (GA) was the first to appear in this field because of the revolutionary research work by Holland [17]. Since then, a number of genetic and swarm optimization strategies have been evolved by various scientists and mathematicians and a significant percentage of them are being used to solve many of the real life problems of practical interest.

One recent development on the traditional GA is the inclusion of self organizing capability of the new random immigrants which are added to the population to increase the potential range. This algorithm is actually inspired by previously proposed Random Immigrants Genetic Algorithm (RIGA) [18] which obeys the concept of flux of immigrants in any biological population. This flux of immigrants is mainly responsible for enhancing the genetic diversity level of the population and accordingly helps to escape it from the local optima points.

It has been reported in literature that systems with several interacting constituents may show one kind of self organizing behaviour. Systems with this self organizing criticality (SOC) are characterized by the phenomenon that even without any control action from the external world, they may self organize themselves in a critical state [19]. The best feature of SOC is perhaps its capability of avoiding situation where an individual species gets entrapped into a local minimum point in the fitness landscape. As a matter of fact, researchers have tried to introduce the concept of SOC in optimization process. The idea of SOC has been incorporated in the RIGA scheme developing a new idea of evolutionary optimization, called as Self-organizing Random Immigrants Genetic Algorithm (SORIGA) [19].

Connection between genetic evolution and SOC has been explored by means of a simple simulation model, known as Bak-Sneppen model [20]. In one dimensional version of this model, species are placed on a circle and are assigned with random fitness value. After the end of each evolution, the fitness value of the individual with lowest fitness in the current population is replaced by random value along with that of the individuals located to its immediate right and left position. As a result of these substitutions of fitness values, average fitness of the entire population starts improving till it ceases to increase indicating the critical state.

SORIGA differs from RIGA in terms of the number of individuals getting replaced in current replacement event and the way in which each individual is selected into the new population [19]. SORIGA is in fact associated with a new parameter, known as replacement rate, which indicates the number of individual members replaced during the current replacement event. Selection phase in SORIGA is modified slightly as compared to conventional GA. If an individual is not involved in the current replacement event, the new individual is selected from the main population. Otherwise, using a standard selection scheme, it is selected from the subpopulation consisting of individuals getting replaced in any replacement event.

\subsection{Design of Powers-of-two FIR Filter using SORIGA}

This paper focuses on the design of powers-of-two FIR filters with the aid of SORIGA. With reference to equation (3), this optimization mechanism helps in locating the mask coefficients $b_{i j}$ which form the basis in constructing the filter coefficients $h_{i}$. Collection of these mask coefficients for the entire set of filter coefficients has been encoded into a single vector $\mathbb{C}$ which acts as a potential chromosome in our design process. Each element of the vector $\mathbb{C}$ is considered as an individual gene which undergoes various genetic operations through evolutions. Fitness of individual chromosome has been calculated based upon the proximity of the designed response with that of the ideal low-pass behaviour. In our work, we have considered the minimax criterion 
of error function to evaluate single chromosome. This has its mathematical illustrations as follows:

$$
\begin{aligned}
& \varepsilon_{p b}(i)=\max _{k}\left\{\left|1-H_{i}(k)\right|\right\} ; 0 \leq k \leq \frac{\omega_{p} N}{2 \pi} \\
& \varepsilon_{t b}(i)=\max _{k}\left\{\left|0.5-H_{i}(k)\right|\right\} ; \quad \frac{\omega_{p} N}{2 \pi} \leq k \leq \frac{\omega_{s} N}{2 \pi} \\
& \varepsilon_{s b}(i)=\max _{k}\left\{\left|H_{i}(k)\right|\right\} ; k>\frac{\omega_{s} N}{2 \pi}
\end{aligned}
$$

The term $H_{i}(k)$ in the above equations identifies the FFT samples of $i^{t h}$ mask set of the designed filter and is related to filter coefficients $h_{i}$ as:

$$
H_{i}(k)=\sum_{i=0}^{L-1} h_{i} e^{-j 2 \pi k n / N}
$$

The maximum of these three error values described in (4), (5) and (6) has been considered as the effective cost functional value for our design purpose which is having the form:

$\varepsilon_{i}=\max \left\{\varepsilon_{p b}(i), \varepsilon_{t b}(i), \varepsilon_{s b}(i)\right\}$

A much better approach for establishing the competence of the evolved chromosome as a mask set of multiplier-less FIR filter can be formulated by assessing the fitness of each chromosome $\varphi(i)$ which is related to cost functional value as:

$$
\varphi\left(\mathbb{C}_{\mathrm{i}}\right)=1 / \varepsilon_{\mathrm{i}}
$$

Entire design process has been elaborated in this section by means of a flow chart as depicted in Figure 1.

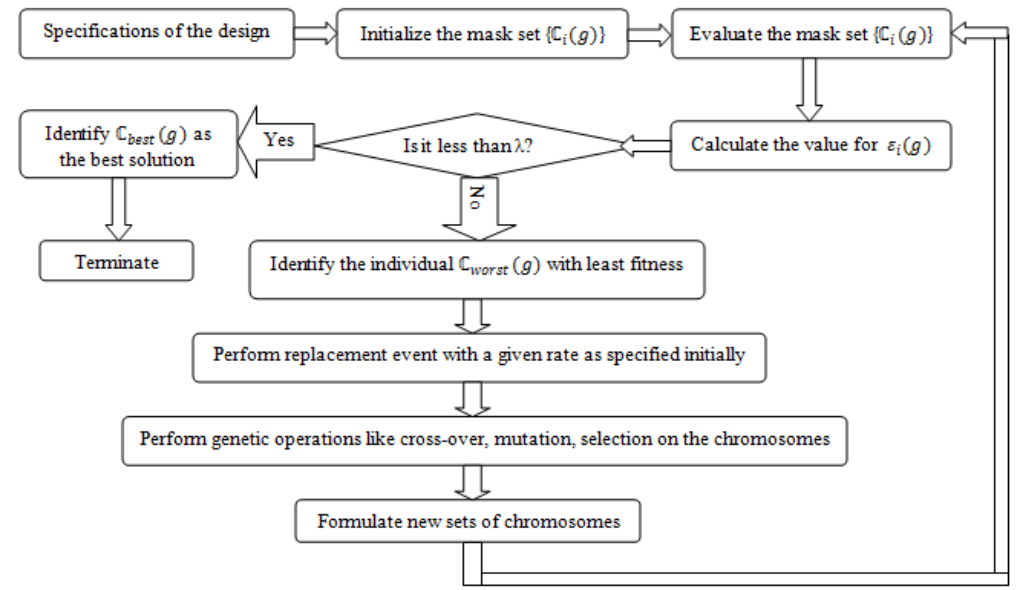

Figure 1. Flow chart of the proposed algorithm for designing multiplier-less powers-of-two FIR filter using Self-organizing Random Immigrants Genetic Algorithm

The flow chart in Figure 1 clearly identifies the various operations as included in our proposed approach for designing powers-of-two FIR filters by means of SORIGA. With reference to Figure 1 , specifications of the design process have been summarized in Table 1 and 2 in the next section. 
Vectors $\mathbb{C}_{\text {best }}(g)$ and $\mathbb{C}_{\text {worst }}(g)$ identify the chromosome, consisting of $\left\lfloor\frac{L}{2}\right\rfloor \cdot \Delta$ number of elements or genes, which yield the maximum fitness (minimum cost) and minimum fitness (maximum cost) respectively amongst the population set $\mathbb{P}$. Number of genes that a single chromosome comprises of depends on the order of the FIR filter and admissible word length of filter coefficients. Terminations of the algorithm is directly associated with the comparison of individual cost function with a predefined threshold $\lambda$.

\section{SimUlATION RESULTS}

This section focuses on our specific achievement in synthesizing multiplier-less low-pass FIR filter with the aid of SORIGA. In connection to this, both binary and CSD representation of the filter coefficients have been taken into our consideration. Selection of different design variables has been listed in Table 1 and 2 below.

Table 1. Specifications of the design process from the perspective of SORIGA

\begin{tabular}{|c|c|}
\hline Parameter & Value \\
\hline Size of the population & 100 \\
\hline Maximum number of iterations & 500 \\
\hline Cross-over probability & 0.5 \\
\hline Mutation probability & 0.01 \\
\hline Replacement rate & 5 \\
\hline Error threshold & $10^{-2}$ \\
\hline
\end{tabular}

Table 2. Specifications of the design process from the perspective of FIR filter

\begin{tabular}{|c|c|c|}
\hline \multirow{2}{*}{ Filter characteristic } & \multicolumn{2}{|c|}{ Name of the filter } \\
\cline { 2 - 3 } & $\mathbf{F}_{\mathbf{1}}$ & $\mathbf{F}_{\mathbf{2}}$ \\
\hline Length & 12 & 36 \\
\hline Word-length of filter coefficient & $8 / 10 / 12 \mathrm{bits}$ & $8 / 10 / 12 \mathrm{bits}$ \\
\hline Pass-band edge frequency & $0.15 \mathrm{rad} / \mathrm{pi}$ & $0.03 \mathrm{rad} / \mathrm{pi}$ \\
\hline Stop-band edge frequency & $0.6 \mathrm{rad} / \mathrm{pi}$ & $0.3 \mathrm{rad} / \mathrm{pi}$ \\
\hline Maximum allowable ripple in pass-band & $1 \mathrm{~dB}$ & $1 \mathrm{~dB}$ \\
\hline Minimum allowable attenuation in stop-band & $50 \mathrm{~dB}$ & $100 \mathrm{~dB}$ \\
\hline
\end{tabular}

In this section, we have demonstrated two design examples of different specifications as outlined in Table 2 and henceforth refer to them as $F_{1}$ and $F_{2}$ respectively. In this context, individual powers-of-two coefficients of $F_{1}$ and $F_{2}$ have been optimized by means of SORIGA with design parameters as in Table 1. Frequency response and impulse response of the proposed SORIGAoptimized low-pass FIR filters has been subsequently presented in Figure 2 and 3 respectively. 
International Journal of Artificial Intelligence \& Applications (IJAIA), Vol. 4, No. 4, July 2013

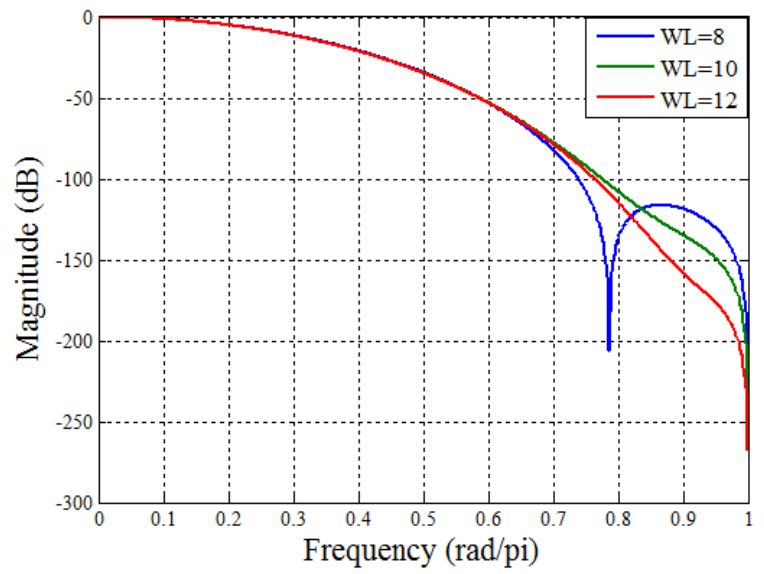

(a)

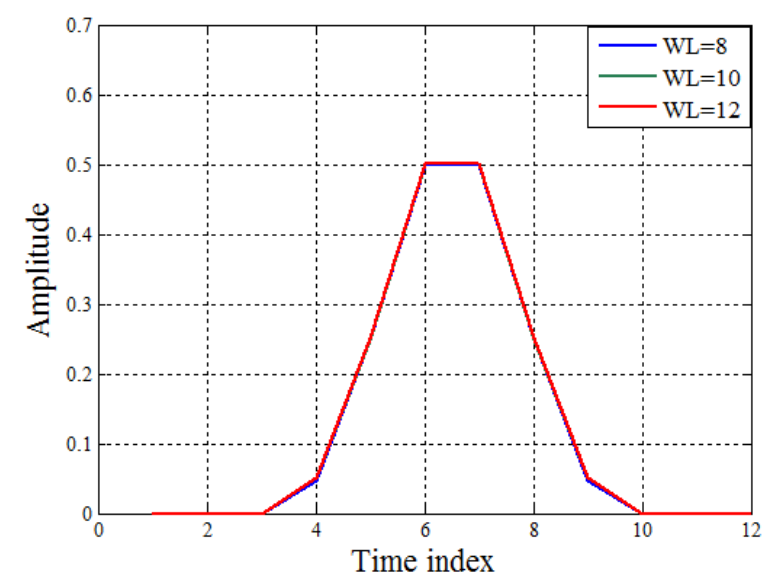

(b)

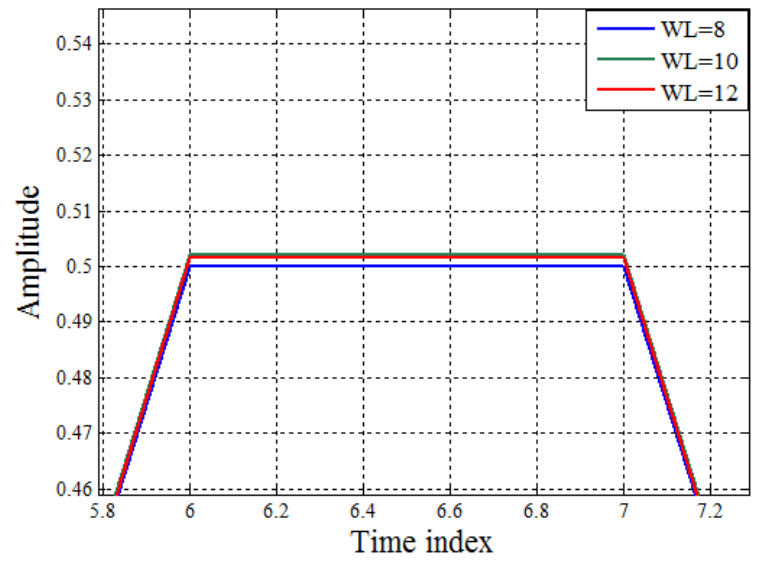

(c)

Figure 2. (a) Frequency response (b) Impulse response (c) Impulse response (zoom in) of $F_{1}$ 
International Journal of Artificial Intelligence \& Applications (IJAIA), Vol. 4, No. 4, July 2013

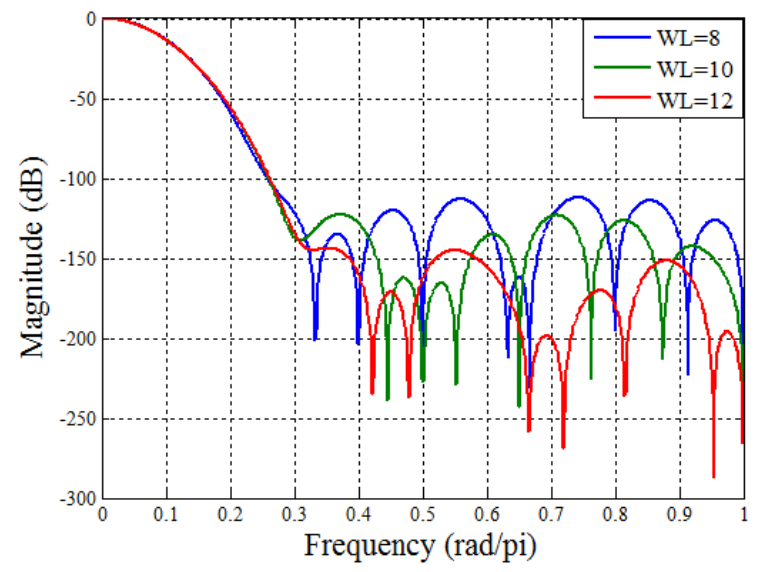

(a)

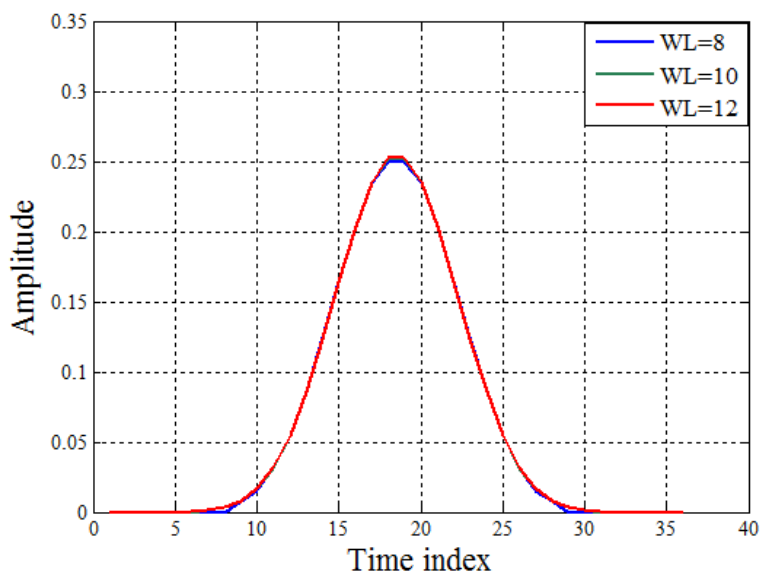

(b)

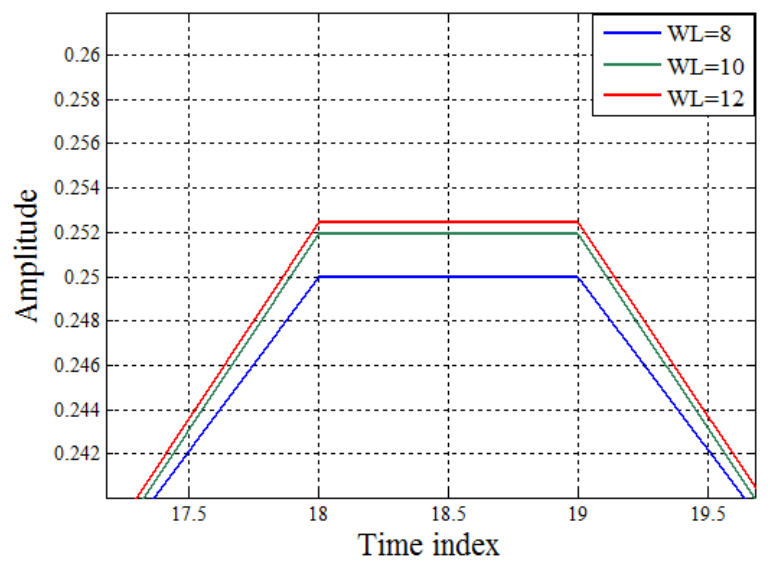

(c)

Figure 3. (a) Frequency response (b) Impulse response (c) Impulse response (zoom in) of $\mathrm{F}_{2}$ From Figure 2(a) and 3(a), it can be unambiguously observed that the designed filters satisfactorily meet the required specifications in the frequency domain. Additionally, its performance remains fairly insensitive to the choice of word-length in the pass-band and the transition-band of current interest. However, on an average, higher word-length leads to more attenuation in the stop-band region of operation. This has been substantiated by listing the 
International Journal of Artificial Intelligence \& Applications (IJAIA), Vol. 4, No. 4, July 2013

attenuation values of $\mathrm{F}_{2}$ at some discrete frequency points in the stop-band region as shown in Table 3 below. In view of impulse response, an interesting feature of the filter may be observed. The impulse response of the designed filter does not contain any side lobe which seems to be an attractive feature of the proposed scheme. In this perspective, higher word-length value results in slightly larger peak of impulse response as seen in Figure 2(c) and 3(c) respectively. Tap coefficients of $F_{1}$ and $F_{2}$ have been summarized in Table 4 and 5 respectively.

Table 3. Stop-band attenuation (in $\mathrm{dB}$ ) of $\mathrm{F}_{2}$

\begin{tabular}{|c|c|c|c|c|c|c|c|}
\hline \multirow{2}{*}{ WL } & \multicolumn{7}{|c|}{ Frequency points (in rad/pi) } \\
\cline { 2 - 8 } & $\mathbf{0 . 3 5}$ & $\mathbf{0 . 4 5}$ & $\mathbf{0 . 5 5}$ & $\mathbf{0 . 6 5}$ & $\mathbf{0 . 7 5}$ & $\mathbf{0 . 8 5}$ & $\mathbf{0 . 9 5}$ \\
\hline 8 & 141.1 & 120.1 & 113.4 & 161.4 & 112.5 & 114.1 & 126.6 \\
\hline 10 & 125.3 & 183.7 & 201.7 & 190.4 & 143.6 & 139.4 & 148.9 \\
\hline 12 & 143.8 & 171.2 & 145.2 & 190.8 & 178.6 & 158.4 & 218.8 \\
\hline
\end{tabular}

Table 4. Tap coefficients of $\mathrm{F}_{1}(\mathrm{WL}=10)$

\begin{tabular}{|c|c|c|c|}
\hline \multicolumn{2}{|c|}{ Tap number } & Binary presentation & CSD presentation \\
\hline $0,1,2$ & $11,10,9$ & 0 & 0 \\
\hline 3 & 8 & $2^{-5}+2^{-6}+2^{-8}$ & $2^{-4}-2^{-6}+2^{-8}$ \\
\hline 4 & 7 & $2^{-2}$ & $2^{-2}$ \\
\hline 5 & 6 & $2^{-1}+2^{-9}$ & $2^{-1}+2^{-9}$ \\
\hline
\end{tabular}

Table 5. Tap coefficients of $\mathrm{F}_{2}(\mathrm{WL}=10)$

\begin{tabular}{|c|c|c|c|}
\hline \multicolumn{2}{|c|}{ Tap number } & $\begin{array}{c}\text { Binary } \\
\text { presentation }\end{array}$ & CSD presentation \\
\hline $0,1,2,3,4,5$ & $35,34,33,32,31,30$ & 0 & 0 \\
\hline 6 & 29 & $2^{-9}$ & $2^{-9}$ \\
\hline 7 & 28 & $2^{-8}$ & $2^{-8}$ \\
\hline 8 & 27 & $2^{-7}$ & $2^{-7}$ \\
\hline 9 & 26 & $2^{-6}+2^{-9}$ & $2^{-6}+2^{-9}$ \\
\hline 10 & 25 & $2^{-5}$ & $2^{-5}$ \\
\hline 11 & 24 & $2^{-5}+2^{-6}+2^{-7}$ & $2^{-4}-2^{-7}$ \\
\hline 12 & 23 & $2^{-4}+2^{-6}+2^{-7}$ & $2^{-3}-2^{-5}-2^{-7}$ \\
\hline 13 & 22 & $2^{-4}+2^{-5}+2^{-6}+2^{-7}+2^{-}$ & $2^{-3}-2^{-9}$ \\
\hline 14 & 21 & $2^{-3}+2^{-5}+2^{-7}$ & $2^{-3}+2^{-5}+2^{-7}$ \\
\hline 15 & 20 & $2^{-3}+2^{-4}+2^{-6}$ & $2^{-2}-2^{-4}+2^{-6}$ \\
\hline 16 & 19 & $2^{-3}+2^{-4}+2^{-5}+2^{-6}$ & $2^{-2}-2^{-6}$ \\
\hline 17 & 18 & $2^{-2}+2^{-9}$ & $2^{-2}+2^{-9}$ \\
\hline
\end{tabular}

Based on the specifications of the multiplier-less FIR filter given above, hardware efficiency of the designed filter can be best evaluated in terms of few performance parameters like Total Power of Two (TPT) terms, Total number of Adders (TA) categorized into Multiplier Adders (MA) and Structural Adders (SA) and Total Delay Flip-flops (TDF) classified into Multiplier Delay Flipflops (MDF) and Structural Delay Flip-flops (SDF). In this regard, hardware cost of $F_{1}$ and $F_{2}$ have been investigated in terms of these parameters for three different values of word-length. Subsequent outcomes considering both the binary and CSD representation have been outlined in Table 6 and 7. 
International Journal of Artificial Intelligence \& Applications (IJAIA), Vol. 4, No. 4, July 2013

Table 6. Hardware cost of SORIGA-optimized low-pass FIR filter with binary coefficients

\begin{tabular}{|c|c|c|c|c|c|c|}
\hline \multirow{2}{*}{ Parameter } & \multicolumn{3}{|c|}{$\mathbf{F}_{\mathbf{1}}$} & \multicolumn{3}{c|}{$\mathbf{F}_{\mathbf{2}}$} \\
\cline { 2 - 7 } & $\mathbf{W L = 8}$ & $\mathbf{W L = 1 0}$ & $\mathbf{W L}=\mathbf{1 2}$ & $\mathbf{W L = 8}$ & $\mathbf{W L = 1 0}$ & $\mathbf{W L = 1 2}$ \\
\hline TPT & 8 & 12 & 20 & 42 & 60 & 84 \\
\hline MA & 2 & 6 & 14 & 22 & 36 & 58 \\
\hline SA & 11 & 11 & 11 & 35 & 35 & 35 \\
\hline TA=MA+SA & 13 & 17 & 25 & 57 & 71 & 93 \\
\hline MDF & 28 & 62 & 152 & 208 & 350 & 612 \\
\hline SDF & 11 & 11 & 11 & 35 & 35 & 35 \\
\hline TDF=MDF+SDF & 39 & 73 & 163 & 243 & 385 & 647 \\
\hline
\end{tabular}

Table 7. Hardware cost of SORIGA-optimized low-pass FIR filter with CSD coefficients

\begin{tabular}{|c|c|c|c|c|c|c|}
\hline \multirow{2}{*}{ Parameter } & \multicolumn{3}{|c|}{$\mathbf{F}_{\mathbf{1}}$} & \multicolumn{3}{|c|}{$\mathbf{F}_{\mathbf{2}}$} \\
\cline { 2 - 7 } & $\mathbf{W L = 8}$ & $\mathbf{W L = 1 0}$ & $\mathbf{W L = 1 2}$ & $\mathbf{W L = 8}$ & $\mathbf{W L = 1 0}$ & $\mathbf{W L = 1 2}$ \\
\hline TPT & 8 & 12 & 18 & 36 & 46 & 66 \\
\hline MA & 2 & 6 & 12 & 16 & 22 & 40 \\
\hline SA & 11 & 11 & 11 & 35 & 35 & 35 \\
\hline TA=MA+SA & 13 & 17 & 23 & 51 & 57 & 75 \\
\hline MDF & 26 & 60 & 126 & 168 & 256 & 474 \\
\hline SDF & 11 & 11 & 11 & 35 & 35 & 35 \\
\hline TDF=MDF+SDF & 37 & 71 & 137 & 203 & 291 & 509 \\
\hline
\end{tabular}

Looking at the above tables, it can be well inspected that except the order-dependent parameters like SA and SDF; word-length of FIR coefficients is having significant impact on the rest of the parameters of present concern. More specifically, higher value of WL signifies larger TPT, MA (as well as TA) and MDF (as well as TDF) and thus results in huge hardware cost. However, because of its canonic structure, CSD representation of filter coefficients outperforms the binary architecture or exhibits comparable performance in terms of the hardware cost incurred. Percentage improvement in resultant hardware cost from CSD representation has been included in Table 8 for both $F_{1}$ and $F_{2}$ respectively.

Table 8. Percentage improvement resulting from CSD representation

\begin{tabular}{|c|c|c|c|c|c|c|}
\hline \multirow{2}{*}{ Parameter } & \multicolumn{3}{|c|}{$\mathbf{F}_{\mathbf{1}}$} & \multicolumn{3}{c|}{$\mathbf{F}_{\mathbf{2}}$} \\
\cline { 2 - 7 } & $\mathbf{W L = 8}$ & $\mathbf{W L = 1 0}$ & $\mathbf{W L = 1 2}$ & $\mathbf{W L}=\mathbf{8}$ & $\mathbf{W L = 1 0}$ & $\mathbf{W L = 1 2}$ \\
\hline TPT & - & - & $10 \%$ & $14.29 \%$ & $23.33 \%$ & $21.43 \%$ \\
\hline TA & - & - & $8 \%$ & $10.53 \%$ & $19.72 \%$ & $19.35 \%$ \\
\hline TDF & $5.13 \%$ & $2.74 \%$ & $15.95 \%$ & $16.46 \%$ & $24.42 \%$ & $21.33 \%$ \\
\hline
\end{tabular}

It is worth mentioning that the values of these performance parameters are very much dependent on the order of the designed FIR filter which, in practice, is adjusted judiciously in order to satisfy the given requirement. As a matter of fact, hardware cost associated with any powers-of-two FIR filter may only be evaluated properly when these parameters are calculated per unit length of the filter. Such an analysis has been carried out in this work and the corresponding outcome has been presented in Figure 4 through 6 below. More specifically, Figure 4 and 5 consider $F_{1}$ and $F_{2}$ respectively for our analysis; while Figure 6 includes few state-of-the-art powers-of-two FIR filters for the purpose of establishing the supremacy of SORIGA-optimized design strategy. Results depicted in Figure 6 have been obtained by comparing the performance of the existing 
International Journal of Artificial Intelligence \& Applications (IJAIA), Vol. 4, No. 4, July 2013

multiplier-less filters with the proposed technique using a word-length value of 10 for each coefficient.

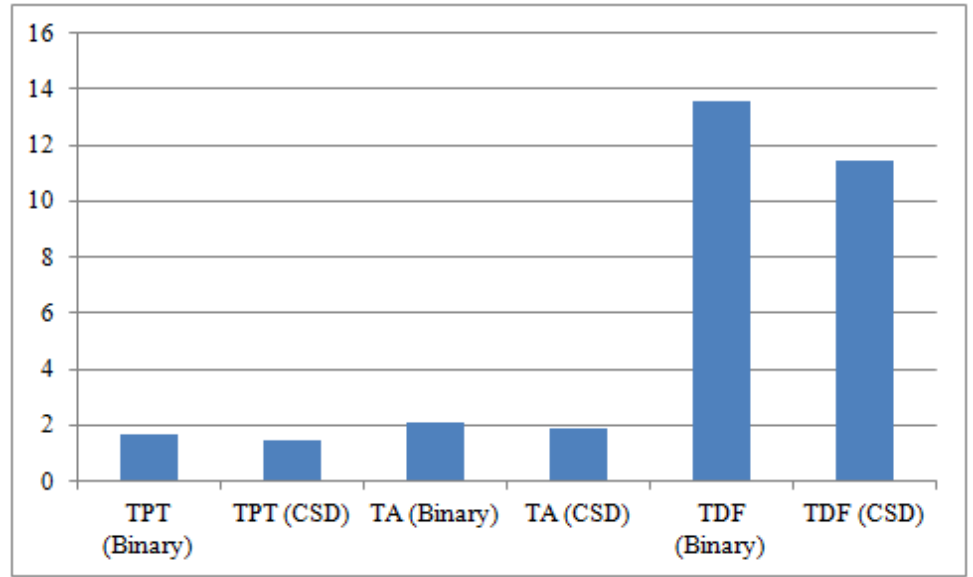

Figure 4. Comparison in terms of hardware cost per unit length between binary and CSD representation for the coefficients of $F_{1}(\mathrm{WL}=12)$

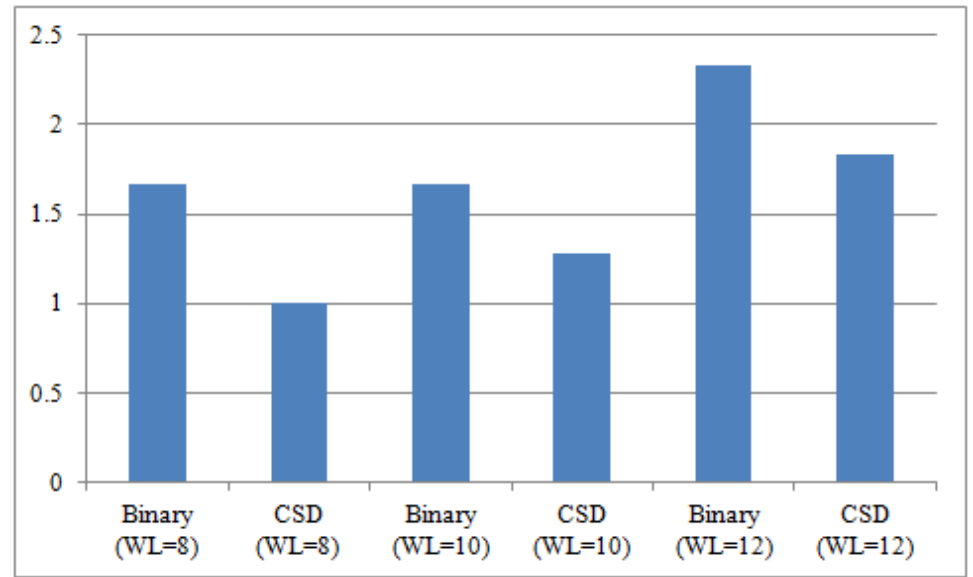

(a)

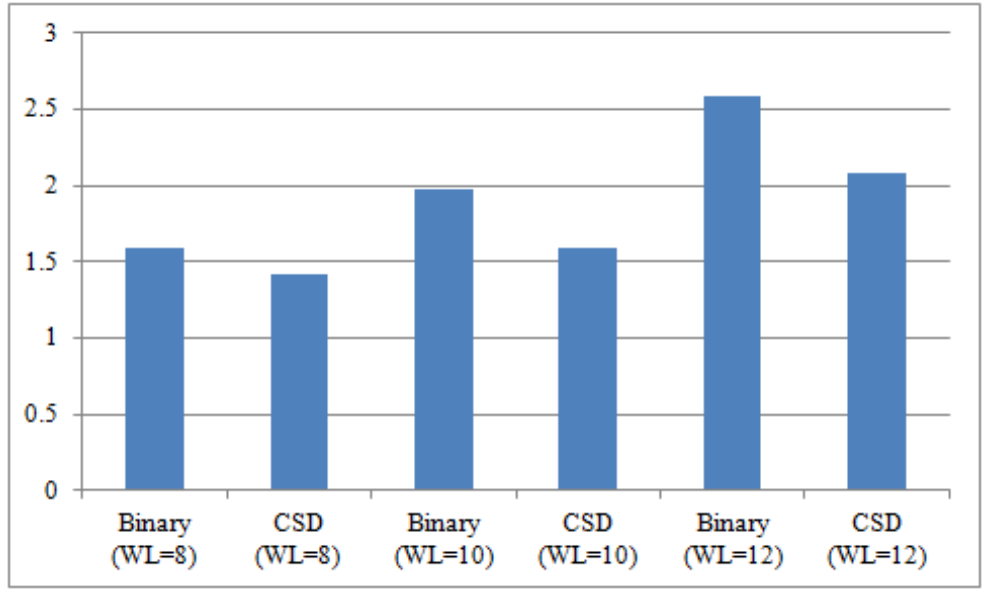

(b) 
International Journal of Artificial Intelligence \& Applications (IJAIA), Vol. 4, No. 4, July 2013

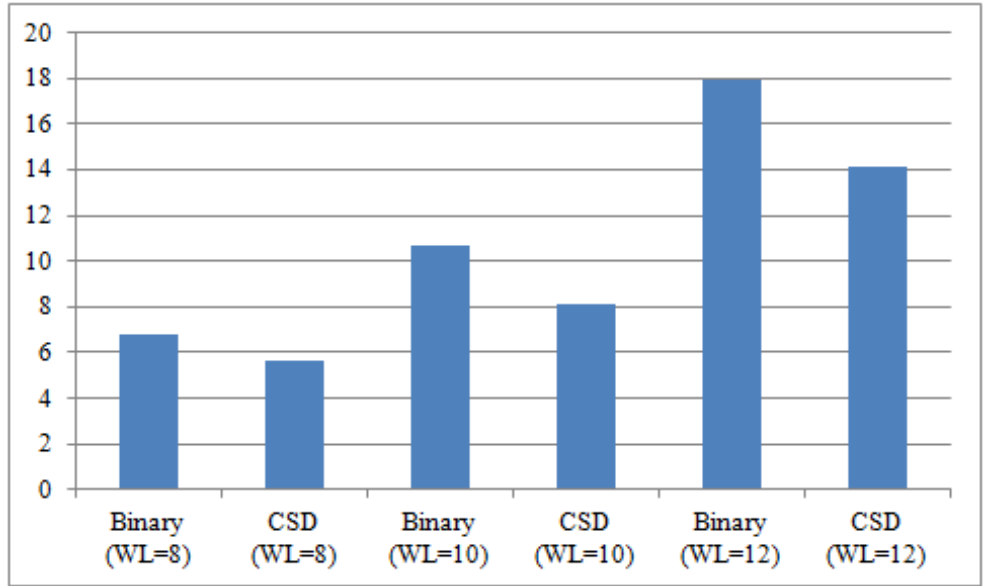

(c)

Figure 5. Comparison in terms of (a) TPT (b) TA (c) TDF per unit length between binary and CSD representation for the coefficients of $\mathrm{F}_{2}$

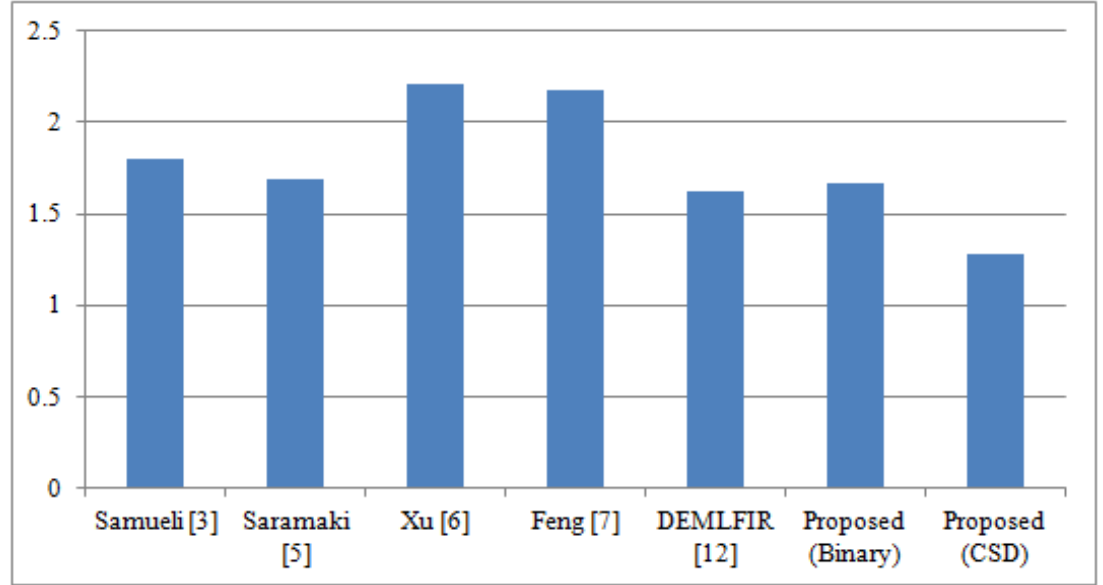

(a)

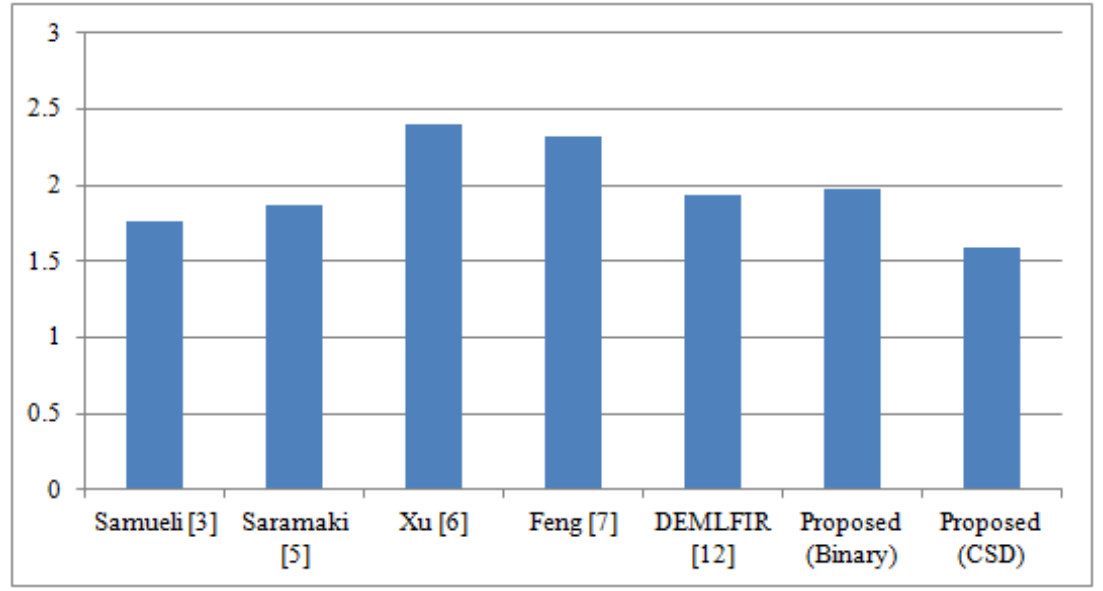

(b) 
International Journal of Artificial Intelligence \& Applications (IJAIA), Vol. 4, No. 4, July 2013

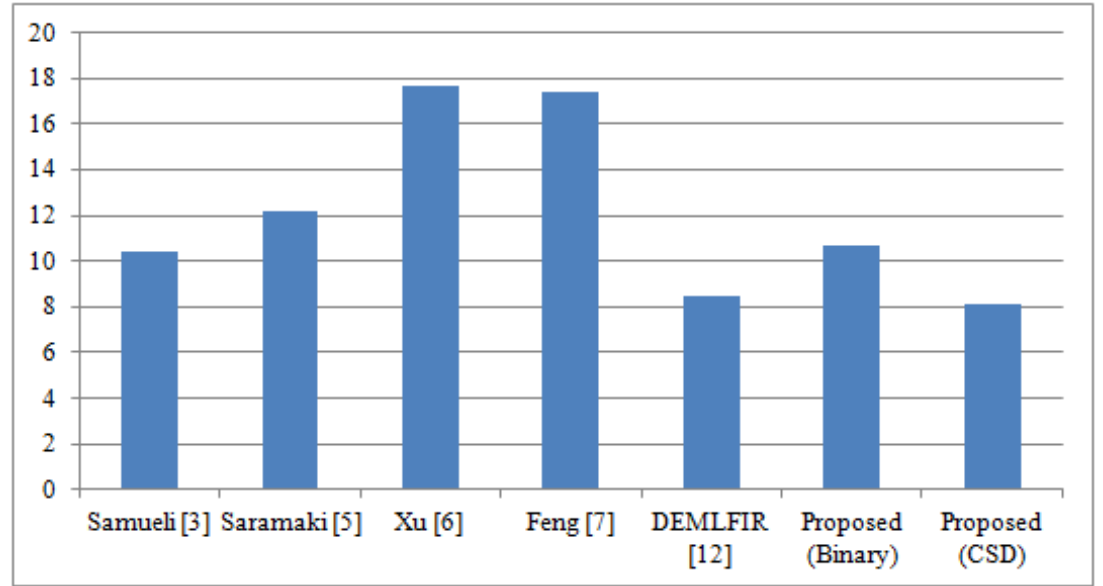

(c)

Figure 6. Comparison in terms of (a) TPT (b) TA (c) TDF per unit length between the proposed $\left(\mathrm{F}_{2}\right)$ and state-of-the-art multiplier-less FIR filter

Looking at Figure 6, it can be well apprehended that CSD-based representation yields less hardware cost per unit length as compared to the design in [3], [5], [6] and [7]. As far as the design in [12] is concerned, CSD-based representation outperforms by a significant margin in terms of TPT and TA per unit length and performs almost similarly in terms of TDF per unit length of the designed filter. In this context, the exact numerical improvement as achieved in the proposed design has been provided in Table 9 for CSD representation.

Table 9. Percentage improvement of CSD-representation over state-of-the-art design techniques

\begin{tabular}{|c|c|c|c|}
\hline \multirow{2}{*}{ Algorithm } & \multicolumn{3}{|c|}{ Improvement in hardware cost per unit length } \\
\cline { 2 - 4 } & TPT & TA & TDF \\
\hline Samueli [3] & $29 \%$ & $10.06 \%$ & $22.28 \%$ \\
\hline Saramaki [5] & $24.38 \%$ & $14.98 \%$ & $33.78 \%$ \\
\hline Xu [6] & $42.28 \%$ & $33.85 \%$ & $54.28 \%$ \\
\hline Feng [7] & $41.27 \%$ & $31.88 \%$ & $53.66 \%$ \\
\hline DEMLFIR [12] & $21.16 \%$ & $18.02 \%$ & $4.72 \%$ \\
\hline
\end{tabular}

\section{CONCLUSIONS}

In this communication, design of a multiplier-less low-pass FIR filter has been carried out by means of Self-organizing Random Immigrants Genetic Algorithm. Tap coefficients of the designed filter have been encoded as sums and/or differences of powers-of-two. Hardware complexity of the proposed design has been computed through the incorporation of a number of performance parameters. Furthermore, the design efficiency of the proposed filter has been compared with a number of existing design techniques. Results reported in the paper establish the supremacy of the proposed design. The work presented in this paper may further be extended by implementing the filters on a real time hardware chip and calculating the actual memory area consumed by each of these structures.

\section{REFERENCES}

[1] Mitra, S. K. (2001) Digital Signal Processing: A Computer-based Approach, 2nd Edition, McGraw Hill. 
International Journal of Artificial Intelligence \& Applications (IJAIA), Vol. 4, No. 4, July 2013

[2] Antoniou, A. (2001) Digital Filters: Analysis, Design and Applications, McGraw-Hill.

[3] Samueli, H. (1989) “An Improved Search Algorithm for the Design of Multiplier-less FIR Filters with Power-of-Two Coefficients”, IEEE Transactions on Circuits and Systems, Vol. 36, No. 7, pp. 10441047.

[4] Chen, C. \& Willson, A. N. (1999) "A Trellis Search Algorithm for the Design of FIR Filters with Signed-Powers-of-Two Coefficients", IEEE Transactions on Circuits and Systems-II: Analog and Digital Signal Processing, Vol. 46, No. 1, pp. 29-39.

[5] Kaakinen, J. Y. \& Saramaki, T. (2001) "A Systematic Algorithm for the Design of Multiplierless FIR Filters", Proc. 2001 IEEE International Symposium on Circuits and Systems, Vol. 2, pp. 185-188.

[6] Xu, F., Chang, C. H. \& Jong, C. C. (2007) "Design of Low-Complexity FIR Filters Based on SignedPowers-of-Two Coefficients with Reusable Common Subexpressions", IEEE Transactions on Computer Aided Design of Integrated Circuits and Systems, Vol. 26, No. 10, pp. 1898-1907.

[7] Feng, Z. G. \& Teo, K. L. (2008) "A Discrete Filled Function Method for the Design of FIR Filters With Signed-Powers-of-Two Coefficients”, IEEE Transactions on Signal Processing, Vol. 56, No. 1, pp. 134-139.

[8] Lim, Y. C. \& Parker, S. R. (1983) "FIR Filter Design over a Discrete Powers-of-Two Coefficient Space", IEEE Transactions on Acoustic, Speech, Signal Processing, Vol. ASSP-31, No. 3, pp. 583591.

[9] Li, D., Song, J. \& Lim, Y. C. (1993) "A Polynomial-Time Algorithm for Designing Digital Filters with Power-of-Two Coefficients”, Proc. 1993 IEEE International Symposium for Circuits and Systems, Vol. 1, pp. 84-87.

[10] Gentili, P., Piazza, F. \& Uncini, A. (1995) "Efficient Genetic Algorithm Design for Power-of-two FIR Filters", Proc. IEEE International Symposium on Circuits and Systems, Vol. 2, pp. 1268-1271.

[11] Ahmad, S. U. \& Antoniou, A. (2006) "Cascade-form Multiplierless FIR Filter Design Using Orthogonal Genetic Algorithm”, Proc. 2006 IEEE International Symposium on Signal Processing and Information Technology, pp. 932-937.

[12] Chandra, A. \& Chattopadhyay, S. "A Novel Approach for Coefficient Quantization of Low-pass Finite Impulse Response Filter using Differential Evolution Algorithm”, Signal, Image and Video Processing, DOI: 10.1007/s11760-012-0359-4.

[13] Chandra, A. \& Chattopadhyay, S. (2011) "A Novel Self-Adaptive Differential Evolution Algorithm for Efficient Design of Multiplier-less Low-pass FIR Filter", Proc. 2nd International Conference on Sustainable Energy and Intelligent System, pp. 733-738.

[14] Chandra, A. \& Chattopadhyay, S. (2011) "Selection of Computationally Efficient Mutation Strategy of Differential Evolution Algorithm for the Design of Multiplier-less Low-pass FIR Filter”, Proc. 14th International Conference on Computer and Information Technology, pp. 274-279.

[15] Avizienis, A. (1961) "Signed-digit Number Representations for Fast Parallel Arithmetic", IRE Transactions on Electronic Computers, Vol. EC-10, pp. 389-400.

[16] Hewlitt, R. M. \& Swartzlander, E. S. (2000) "Canonical Signed Digit Representation for FIR Digital Filters”, Proc. 2000 IEEE Workshop on Signal Processing Systems (SiPS 2000), pp. 416-426.

[17] Holland, J. H. (1975) Adaptation in Natural and Artificial Systems, University of Michigan Press, Ann Arbor.

[18] Manner, R., Manderick, B. (eds.) (1992) Parallel Problem Solving from Nature, 2, Amsterdam: North-Holland.

[19] Tinos, R. \& Yang, S. (2007) “A Self-organizing Random Immigrants Genetic Algorithm for Dynamic Optimization Problems", Genetic Programming and Evolvable Machines, Vol. 8, No.3, pp. 255-286.

[20] Bak, P. (1997) How Nature Works: The Science of Self-organized Criticality, Oxford University Press. 